TRANSACTIONS OF THE

AMERICAN MATHEMATICAL SOCIETY

Volume 192, 1974

\title{
VAN KAMPEN'S THEOREM FOR $n$-STAGE COVERS
}

\author{
BY \\ J.C. CHIPMAN(1)
}

\begin{abstract}
A version of van Kampen's theorem is obtained for covers whose members do not share a common point and whose pairwise intersection need not be connected.
\end{abstract}

Introduction. One of the principal tools in the computation of fundamental groups has been van Kampen's theorem, which relates the fundamental group of a space to the fundamental groups of the members of a cover of that space. Previous formulations of this result have either been of an algorithmic nature as were the original versions of van Kampen [8] and Seifert [6] or of an algebraic nature such as Crowell's [2] in which he considered the problem from the point of view of the fundamental group functor commuting with the direct limit. With all of these the typical restrictions on the cover have been (a) closed under pairwise intersection, (b) all members connected and, (c) the existence of a common point among all the members of the cover.

The intent of this paper will be to consider the problem for a more general class of covers and will reflect the two concerns of the earlier works, algorithmic and algebraic. From the algebraic point of view it is hoped that the consideration of more general covers will permit a better understanding of the way that a cover decomposes the algebraic information that is represented by the fundamental group of the space. On the other hand from an algorithmic or practical view, versions of the van Kampen theorem for a wider class of covers will permit greater flexibility and facility in the computation of the fundamental groups. These techniques will not only simplify earlier computations such as in [4], but will obtain some new results as in [1].

The setting for the paper will be the simplicial category. A collection of subcomplexes of the connected complex $K$ whose union contains $K$ will be called a cover of $K$. Since we are interested in speaking about the fundamental groups of the members of a cover $\Phi=\left\{K_{\alpha}: \alpha \in \Lambda\right\}$, we shall assume that each of the $K_{\alpha}$ is connected although this is not necessary in some of the theorems. Associated with $\Phi$ there will be what is referred to as the completed cover $\Sigma$. $\Sigma$

Received by the editors September 23, 1971 and, in revised form, July 15, 1972 and March 9, 1973.

AMS (MOS) subject classifications (1970). Primary 55A05.

Key words and phrases. Fundamental group, covers, maximal tree, $n$-stage cover, limit of a group system.

(1) Part of this material was included in the author's Ph.D. dissertation submitted to Dartmouth College and supervised by Professor Edward M. Brown.

Copyright $\odot$ 1974, American Mathematical Society 
will consist of those subcomplexes $A$ such that either (a) $A=K_{\alpha}$ for some $\alpha \in \Lambda$, or (b) $A$ is a path component of $A_{1} \cap A_{2}$ where $A_{1}, A_{2} \in \Sigma$.

The paper is divided into three sections. $\$ 1$ will consider the problem for arbitrary covers and develop the general approach that will be taken. Briefly this will be through the introduction of a new functor $\Gamma$ from the category of simplicial complexes to the category of groups. It will be shown that the functor $\Gamma$ reflects the simplicial structure more completely than the functor $\pi_{1}$ permitting a direct solution to the problem. The functor $\Gamma$ will be related to $\pi_{1}$ to indicate the possibilities for a solution for that functor. In $\$ 2$ a smaller class of covers will be considered. For this class of covers a solution for the functor $\pi_{1}$ will be given which is both computationally simple and algebraically interesting. In $\$ 3$ the class of covers considered in $\$ 2$ will be generalized and a solution given.

1. Let $K$ be a complex and $\Sigma$ a completed cover of $K$; the members of $\Sigma$ and the inclusion maps between them form a system whose limit is $K$. The optimal result would be to observe that this fact remains true when the functor $\pi_{1}$ is applied, but this is not the case, since $\pi_{1}$ fails to reflect completely the simplicial structure of the complex. As a starting point we consider a functor from the category of simplicial complexes to the category of groups which avoids this difficulty. Later this will facilitate the consideration of the original problem for the functor $\pi_{1}$. Let $K$ be any simplicial complex with a chosen orientation for its simplexes. We define $\Gamma(K)=\left|K^{1}: \partial K^{2}\right|$. That is to say $\Gamma(K)$ is a group whose presentation consists of generators in one-to-one correspondence with the oriented 1-simplexes of $K$ and of relations in one-to-one correspondence with the oriented boundaries of the oriented 2-simplexes of $K$. Given a simplicial map $\varphi: K \rightarrow L$, we can associate a homomorphism $\bar{\varphi}:\left|K^{1}:\right| \rightarrow\left|L^{1}:\right|$ which will induce a homomorphism between the factor groups, $\Gamma(\varphi): \Gamma(K) \rightarrow \Gamma(L)$. Defined in such a way, $\Gamma$ is a functor. $\Gamma$ can also be extended to a functor on the category of simplicial pairs by defining $\Gamma(K, L)$ to be the group whose presentation is that of $\Gamma(K)$ with the relation set enlarged to include the 1-simplexes of $L . \Gamma(\varphi)$ : $\Gamma\left(K_{1}, L_{1}\right) \rightarrow \Gamma\left(K_{2}, L_{2}\right)$ can then be defined as before.

Suppose now that $\Sigma$ is a completed cover of $K$ by connected subcomplexes. For $A, B \in \Sigma$ such that $A \subseteq B$ let $i_{A B}: \Gamma(A) \rightarrow \Gamma(B)$ and $i_{A}: \Gamma(A) \rightarrow \Gamma(K)$ be the homomorphisms induced by the respective inclusion maps $i: A \rightarrow B$ and $i: A \rightarrow K$. We then have a group system, $\left(\Gamma(A), i_{A B}: \Gamma(A) \rightarrow \Gamma(B) ; A, B \in \Sigma\right)$. Recall that $\left(G, \theta_{A}: \Gamma(A) \rightarrow G ; A \in \Sigma\right)$ is said to be the limit of this system if the following universal property is satisfied: for any group $H$ and family of homomorphisms $\left\{j_{A}: \Gamma(A) \rightarrow H ; A \in \Sigma\right\}$ for which $A \subseteq B$ implies that $j_{A}$ $=j_{B} i_{A B}$; there exists a unique homomorphism $\theta: G \rightarrow H$ such that $j_{A}=\theta \theta_{A}$ for all $A \in \Sigma$. Since $\Gamma$ copies the simplicial structure of the 2-skeleton of $K$, the limit property that holds for simplicial complexes now carries over to the associated groups by a proof that is essentially the same for both. 
Theorem 1.1. $\left(\Gamma(K), i_{A}: \Gamma(A) \rightarrow \Gamma(K) ; A \in \Sigma\right)$ is the limit of the group system $\left(\Gamma(A), i_{A B}: \Gamma(A) \rightarrow \Gamma(B) ; A, B \in \Sigma\right)$.

Proof. The existence of $\theta$ will be considered first. Let $x$ be an arbitrary generator of $\Gamma(K)$. Since the members of $\Sigma$ cover $K$ there is an $A \in \Sigma$ with $x \in A$ and hence a $y \in \Gamma(A)$ with $i_{A}(y)=x$. To obtain $j_{A}=\theta i_{A}$, we are then forced to define $\bar{\theta}(x)=j_{A}(y)$. Since $\Sigma$ is a completed cover it is not hard to check that $\bar{\theta}$ is well defined and thereby yields a homomorphism $\bar{\theta}:\left|K^{1}:\right| \rightarrow H$. Let $\eta:\left|K^{1}:\right| \rightarrow \Gamma(K)$ be the factor homomorphism. We can obtain $\theta: \Gamma(K) \rightarrow H$ such that $\bar{\theta}=\theta \eta$ provided that $\operatorname{ker} \eta \subseteq \operatorname{ker} \bar{\theta}$. Recall now that a typical generator of $\operatorname{ker} \eta$ is $\partial \sigma$ where $\sigma$ is a 2-simplex of $K$. Since $\sigma \in A$ for some $A \in \Sigma$, if $\partial \sigma=x_{1} x_{2} x_{3}$ then in $\Gamma(A)$ we have for the corresponding elements $y_{1} y_{2} y_{3}=1$. Therefore $\bar{\theta}(\partial \sigma)=j_{A}\left(y_{1}\right) j_{A}\left(y_{2}\right) j_{A}\left(y_{3}\right)=j_{A}\left(y_{1} y_{2} y_{3}\right)=1 \in H$. So the existence of $\theta: \Gamma(K) \rightarrow H$ is established; its uniqueness and compatibility with the $i_{A}$ and the $j_{A}$ are inherent in its construction. The proof is now complete.

Consider now an arbitrary subcomplex $L$ of $K$. Then as before we can consider induced homomorphisms $k_{A B}: \Gamma(A, A \cap L) \rightarrow \Gamma(B, B \cap L) \quad k_{A}: \Gamma(A, A \cap L)$ $\rightarrow \Gamma(K, L) ; A, B \in \Sigma$ and $A \subseteq B$. Indeed Theorem 1.1 holds in this more general setting as well; one need only check that a member of $\Gamma(K, L)$ corresponding to a 1-simplex of $L$ is contained in $\operatorname{ker} \bar{\theta}$. But if $x \in L^{1}$ then $x \in A$ for some $A \in \Sigma$. Hence the corresponding element $y=1 \in \Gamma(A, A \cap L)$ and as such $j_{A}(y)=1 \in H$ and so $\bar{\theta}(x)=1 \in H$ also. We have thus proved the following: proved the following:

Corollary 1.2. Let $L \subseteq K$. Then $\left(\Gamma(K, L), k_{A}: \Gamma(A, A \cap L) \rightarrow \Gamma(K, L) ; A \in \Sigma\right)$ is the limit of the group system $\left(\Gamma(A, A \cap L), k_{A B}: \Gamma(A, A \cap L) \rightarrow \Gamma(B B \cap L) ; A\right.$, $B \in \Sigma)$.

Hence the functor $\Gamma$ reflects the simplicial structure of the complex well enough to permit the representation of the $\Gamma$ group of $K$ in terms of the $\Gamma$ groups of the members of the cover of $K$. This reflection is so strong, however, that $\Gamma(K)$ fails to be an invariant of the underlying space of $K$. For example, consider the $\Gamma$ groups for various triangulations of the unit interval. This handicap is overcome by the fact that $\Gamma(K)$ can be related to $\pi_{l}\left(K, v_{0}\right)$. The next series of propositions is devoted to that end.

The well-known theorem which lies at the heart of this whole approach establishes the initial relationship of $\Gamma(K)$ to $\pi_{1}\left(K, v_{0}\right)$. In the terminology we are using its formulation is as follows.

Theorem 1.3. Let $T$ be a maximal tree in $K$. Then $\Gamma(K, T) \approx \pi_{1}\left(K, v_{0}\right)$. The isomorphism assigns to each generator $x$ of $\Gamma(K, T)$ the class of the loop which runs in $T$ from $v_{0}$ to the initial vertex of $x$, along $x$, and then back to $v_{0}$ in $T$ from the terminal vertex of $x$. 
Proof. Cf. [7].

Consider now a more general pair $(K, L)$ where $L$ is a 1-dimensional subcomplex of $K$ which is contained in some maximal tree $T$ of $K$. For each $x \in T-L$, let $C(\partial x)=a_{x} \cup b_{x}$ denote the cone over the boundary of $x$, where $a_{x}$ connects to $x_{0}$ and $b_{x}$ to $x_{1}$, the initial and terminal vertices of $x$ respectively. Form the complex $\tilde{K}=K \cup \cup_{x \in T-L} C(\partial x)$. Let the orientation of $\tilde{K}^{n}$ coincide with that of $K^{n}$ for $n>1$, orient $\tilde{K}^{1} \cap K^{1}$ as in $K^{1}$ and pick any orientation for $\tilde{K}^{1}-K^{1}$ with $\left(b_{x}\right)_{1}=x_{1}$. In $\tilde{K}$ we have several choices for maximal trees. For example, $L \cup \cup_{x \in T-L} C(\partial x)$ is a maximal tree $T_{0}$ in $\tilde{K}$. Then $\Gamma\left(\tilde{K}, T_{0}\right)=\Gamma(K, L)$. On the other hand, we have $T_{1}=T \cup \cup_{x \in T-L} a_{x}$ is also a maximal tree for $\tilde{K}$; this yields $\Gamma\left(\tilde{K}, T_{1}\right)=\Gamma(K, T) *|T-L:|$, the free product of the group $\Gamma(K, T)$ and a free group on the set of 1-simplexes of $T-L$. Theorem 1.3 establishes that $\Gamma(K, L) \approx \Gamma(K, T) *|T-L:|$.

If we choose a vertex $v_{0}$ in $K$ an isomorphism, $\varphi: \Gamma(K, L) \rightarrow \Gamma(K, T)$ * $|T-L:|$ can be given explicitly. Since both of these groups are isomorphic to the fundamental group of $\tilde{K}, \varphi$ can be determined by using Theorem 1.3 with respect to the trees $T_{0}$ and $T_{1}$. In the latter case a generator $x \in K-T$ corresponds to the path $p\left(v_{0}, x_{0}\right)$ in $T$ from $v_{0}$ to $x_{0}$, then along $x$, then back to $v_{0}$ by the path $p\left(x_{1}, v_{0}\right)$ in $T$. In the former case such an $x \in K-T$ corresponds to essentially the same loop described above except whenever an edge $y \in T-L$ is encountered along that path the "bridge" provided by $C(\partial y)$ is taken instead. The sequence of such edges of $T-L$ in $p\left(v_{0}, x_{0}\right)$ determines a word $\alpha\left(v_{0}, x_{0}\right)$ in $|T-L:|$ as does the similar sequence in $p\left(x_{1}, v_{0}\right)$ determine $\alpha\left(x_{1}, v_{0}\right)$. (Of course, if an edge $e$ of $T-L$ is transversed in $p\left(v_{0}, x_{0}\right)$ contrary to its orientation we use $e^{-1}$ in $\alpha\left(v_{0}, x_{0}\right)$.) Thus the loop corresponding to $x \in K-T$ in terms of $T_{0}$ is expressed as the product $\alpha\left(v_{0}, x_{0}\right) x \alpha\left(x_{1}, v_{0}\right)$ in terms of the generating loops using $T_{1}$. A similar remark holds for an $x \in T-L$. In summary we have proved:

Theorem 1.4. Let $L$ be a 1-dimensional subcomplex of $K$ that is contained in a maximal tree $T$. Then $\Gamma(K, L) \approx \Gamma(K, T) *|T-L:|$. Moreover if a vertex $v_{0}$ of $K$ is chosen the isomorphism

$$
\varphi: \Gamma(K, L) \rightarrow \Gamma(K, T) *|T-L:|
$$

is given on the generators of $\Gamma(K, L)$ by

$$
\varphi(x)=\alpha\left(v_{0}, x_{0}\right) x \alpha\left(x_{1}, v_{0}\right) .
$$

Corollary 1.5. For any complex $K$ and maximal tree $T, \Gamma(K) \approx \Gamma(K, T) *|T:|$.

The preceding observation establishes a minimal relationship between the fundamental group of the complex and the fundamental groups of the members of the complex. The possible improvements that are available for particular covers or a particular presentation as given by the choice of the maximal tree will be considered in the next section; the present will conclude with an indication of what the possibilities are at this stage. 
For a given complex $K$ and completed cover $\Sigma$, let $T$ be an arbitrary maximal tree. For each $A \in \Sigma$, let $T(A)$ be a maximal tree in $A$ extending the forest, $T \cap A$. For $\Gamma(K, T)$ we then have a set of generators in one-to-one correspondence with the members of $K^{1}-T$. Among these let $W(T)=\left\{x \in K^{1}\right.$ : for every $A \in \Sigma, x \in A$ implies $x \in T(A)-T\}$. W(T) can be interpreted as those generators of $\Gamma(K, T)$ which fail to be represented as a generator in any of the groups $\Gamma(A, T(A))$ of the members of the cover. Let $N(\Sigma, T)$ be the normal subgroup of $\Gamma(K, T)$ generated by $W(T)$ and let $G(\Sigma, T)$ denote $\Gamma(K, T \cup W(T))$; we then have the short exact sequence:

$$
1 \rightarrow N(\Sigma, T) \rightarrow \Gamma(K, T) \rightarrow G(\Sigma, T) \rightarrow 1 .
$$

Since it is not difficult to show that the images of the groups $\Gamma(A, T(A))$ generate $G(\Sigma, T)$ the above sequence indicates how much of the fundamental group of $K$ can be obtained in terms of the fundamental groups of the members of the cover $\Sigma$. The extreme situation with $N(\Sigma, T)$ isomorphic to $\Gamma(K, T)$ can be realized by taking $K$ to be a 2-complex and $\Sigma$ to be the cover generated by the principal simplexes of $K$.

2. The results of $\$ 1$ imply that the directness of the relationship between the group of the complex and the groups of the cover will depend upon the extent to which a maximal tree for the complex can be constructed from maximal trees chosen for the members of the cover. For example, if $K=K_{1} \cup K_{2}$ with $K_{0}=K_{1} \cap K_{2}$ and each $K_{i}$ is connected then the construction of such a maximal tree for $K$ would be as follows. Choose a maximal tree $T_{0}$ for $K_{0}$ and extend $T_{0}$ to maximal trees $T_{1}$ and $T_{2}$ in $K_{1}$ and $K_{2}$ respectively. Then $T=T_{1} \cup T_{2}$ is a maximal tree for $K$ with $T \cap K_{i}=T_{i}$ for each $i$, and we obtain the usual presentation for $\pi_{1}(K)$ as the free product of $\pi_{1}\left(K_{1}\right)$ and $\pi_{1}\left(K_{2}\right)$ with amalgamated subgroup $\pi_{1}\left(K_{1} \cap K_{2}\right)$. In this section we wish to begin to consider covers which will permit a similar construction of a maximal tree. At the risk of some redundancy we will study a particular case of this class of covers. This will be balanced by the insight provided into the ideas of the more general case to be considered in $\$ 3$ and the suitability of this particular case for most computational applications.

Definition 2.1. A 1-stage cover $\Sigma$ of the complex $K$ consists of two collections $\lambda_{0}, \lambda_{1}$ of subcomplexes of $K$ such that:

(i) $\lambda_{0}$ is a cover of $K$ by connected subcomplexes.

(ii) $\lambda_{1}$ is the set of all path components of pairwise intersections $A_{1} \cap A_{2}$, where $A_{1}, A_{2} \in \lambda_{0}$.

(iii) If $A \in \lambda_{0}$, then $A-\cup \lambda_{1}$ is connected and nonempty.

(iv) The set of path components of $U \lambda_{1}$ is equal to $\lambda_{1}$ (or equivalently, $\lambda_{1}$ consists of disjoint sets).

In such a case, we will regard $\Sigma=\lambda_{0} \cup \lambda_{1}$.

A 1-stage cover is simple enough to provide the basis for a level by level construction of a maximal tree but complex enough to prohibit, in general, the 
choice of a maximal tree whose intersection with each member of the cover will form a maximal tree for that member. This can occur as follows. Given a 1-stage cover $\Sigma$, choose maximal trees for each member of $\lambda_{1}$. By condition (iv) the union of these trees will form a forest in $K$ and as such a forest in each member of $\lambda_{0}$. Now extend these forests to maximal trees in each member of $\lambda_{0}$. The union of these trees will form a 1-dimensional subcomplex $G$ of $K$ whose intersection with each member of $\Sigma$ is a maximal tree, but can fail to be a tree in $K$. For let $A_{1}, A_{2}$ be any two members of $\lambda_{0}$ whose intersection consists of at least two path components $B_{1}$ and $B_{2}$; there will be two paths in $G$ from a vertex in $B_{1}$ to a vertex in $B_{2}$, one using the tree in $A_{1}$ and the other using the tree in $A_{2}$.

This, then, represents the essential problem in constructing a maximal tree for the complex that will provide a presentation for the groups of the complex in terms of the groups of the members of the cover and the inclusion homomorphisms between them. As anticipated by the remarks at the end of $\$ 1$ there will be generators (members of the complement of a maximal tree) of $\pi_{1}(K)$ that cannot be viewed as generators (members of the complement of a maximal tree) of any member of the cover. The aim then becomes to control the occurrence of these generators in such a way that a presentation for the group of the complex is available from the cover itself without the messy chore of going into the simplicial structure and selecting a maximal tree.

In this light we consider the 1-stage cover $\Sigma$ more closely as to the information it contains about the complex $K$. First there are the members of $\Sigma$ themselves and the inclusion maps between them which provide local information about $K$. But in a wider sense the very existence of these inclusion maps provides global information about $K$ since they describe how the members of $\Sigma$ are pasted together to form $K$. To record this information we define a bipartite graph $W_{1}$ with one class of vertices in one-to-one correspondence with the elements of $\lambda_{0}$ and the other in one-to-one correspondence with the elements of $\lambda_{1}$.

The vertex in $W_{1}$ corresponding to $A \in \Sigma$ will be denoted $\bar{A}$. Edges are introduced in one-to-one correspondence with the inclusion maps between the elements of $\Sigma$. The graph $W_{1}$ permits the satisfactory completion of the construction of the maximal tree for $K$ attempted before. Essentially this is because $W_{1}$ is of the same homotopy type as the 1-complex $G$ above. More precisely we have

Proposition 2.2. Let $T\left(W_{1}\right)$ be a maximal tree in $W_{1}$. Then maximal trees $T(K)$ for $K$ and $T(A)$ for each $A \in \Sigma$ can be chosen such that:

(i) For each $A \in \lambda_{1}, T(K) \cap A=T(A)$.

(ii) For each $A \in \lambda_{0}, T(K) \cap A \subseteq T(A)$. Furthermore the set of edges of $T(A)-T(K)$ is in one-to-one correspondence with those edges of the complement of $T\left(W_{1}\right)$ incident to $\bar{A}$.

(iii) For $A \in \lambda_{0}$ and $B \in \lambda_{1}$ such that $B \subset A$, the path in $T(A)$ from a vertex in $B$ to a vertex in $A-\cup \lambda_{1}$ contains at most one edge of $T(A)-T(K)$. 
Proof. As before we begin by choosing maximal trees $T(A)$, for each $A \in \lambda_{1}$. The union of these trees $F$ will form a forest in $K$ by 2.1(iv). Hence $F \cap A$ will be a forest in each $A \in \lambda_{0}$. For each $A \in \lambda_{0}, F \cap A$ is extended to a maximal tree in the following way. Choose a vertex $v(A)$ in $A-\cup \lambda_{1}$. Paths from $v(A)$ to a vertex in each $B \in \lambda_{1}$ contained in $A$ are then chosen. This may be done in such a way that the union of $F \cap A$ and the paths so chosen is a tree in $A$ and each path from $v(A)$ to a $B \subset A$ contains an edge (namely the last one not in $B$ ) not contained in any of the other paths so chosen. This is because $A-\cup \lambda_{1}$ is connected and the members of $\lambda_{1}$ are disjoint. This tree is then extended to a maximal tree $T(A)$ for $A$. We now let $G$ be the union of the $T(A)$ for $A \in \lambda_{0}$. Since the members of $\lambda_{0}$ cover $K, G$ contains all the vertices of $K$. A maximal tree $T(K)$ can be obtained from $G$ by deleting appropriate edges.

The tree $T\left(W_{1}\right)$ will be used to determine which edges of $G$ to delete. For each $A \in \lambda_{0}$ consider the collection of edges $(\bar{B}, \bar{A})$ in $W_{1}$ for those $B$ of $\lambda_{1}$ contained in $A$. By the way that $T(A)$ has been constructed, for each edge $(\bar{B}, \bar{A})$ a corresponding edge of $T(A)-\cup \lambda_{1}$ can be chosen such that every path from a vertex in $B$ to a vertex in $A-\cup \lambda_{1}$ contains that edge and no other edge similarly chosen for some other $(\bar{C}, \bar{A})$ of $W_{1}$. We now obtain $T(K)$ from $G$ by deleting those edges of $G$ which correspond to the complement of $T\left(W_{1}\right)$ in $W_{1}$.

The construction insures that the three conditions will be satisfied if $T(K)$ actually is a maximal tree for $K$. Since $T(K)$ contains all the vertices of $K$, it remains to show that there is exactly one reduced path in $T(K)$ between any two vertices. Since $G$ was connected the existence of paths can be established by finding paths connecting the endpoints of any edge that was deleted from $G$ to obtain $T(K)$. Such an edge corresponds to an edge in $W_{1}$, and the desired path may be constructed using the path in $T\left(W_{1}\right)$ between the endpoints of that edge as a guide in $T(K)$. As for the uniqueness of paths, the construction of the $T(A)$ for $A \in \lambda_{0}$ permits the existence of at most one path in $T(K) \cap A$ from a vertex of a member of $\lambda_{1}$ contained in $A$ to a vertex of $A-\cup \lambda_{1}$. This fact and the unique path property for $T\left(W_{1}\right)$ will yield the uniqueness of path in $T(K)$. Hence $T(K)$ is a maximal tree in $K$, and the proof is complete.

We have then the following procedure for obtaining a presentation for the group of the complex $K$ with respect to a given 1-stage cover $\Sigma$. Form the graph $W_{1}$ and choose a maximal tree $T\left(W_{1}\right)$. By applying Proposition 2.2 and the results of $\$ 1, \Gamma(K, T(K))$ will be given as the limit of a group system which is completely described using the groups $\Gamma(A, T(A)), A \in \Sigma$ and $T\left(W_{1}\right)$ in $W_{1}$. As a group presentation it would take the following form:

Theorem 2.3. Let $K$ be a complex with a 1-stage cover $\Sigma$ whose graph is $W_{1}$. Choose a maximal tree $T\left(W_{1}\right)$ in $W_{1}$. Then $\Gamma(K, T)$ has a group presentation with generators and relations:

Generators. (i) For each $A \in \lambda_{0}$, a set of generators of $\Gamma(A, T(A))$,

(ii) For each edge in the complement of $T\left(W_{1}\right)$ a generator $t_{i}$. 
Relations. (i) For each $A \in \lambda_{0}$, a set of relations of $\Gamma(A, T(A))$ in terms of the generators chosen,

(ii) for each $A \in \lambda_{1}$, a set of relations giving rise to an amalgamated subgroup. The homomorphisms whose images are to be amalgamated are determined by the edges incident to $\bar{A}$ in $W_{1}$. If the edge is in $T\left(W_{1}\right)$ the corresponding homomorphism is simply that induced by inclusion. If the edge is in the complement of $T\left(W_{1}\right)$ the corresponding homomorphism is that induced by inclusion followed by conjugation by the $t_{i}$ that corresponds to that edge.

Proof. The general form of the presentation as that of the limit of a group system arises from applying Corollary 1.2 to $K$ with the maximal tree $T(K)$ chosen according to Proposition 2.2. The groups whose subgroups are being amalgamated are $\Gamma(A, A \cap T(K)), A \in \lambda_{0}$. By Theorem 1.4 each of these $\Gamma(A, A \cap T(K))$ is isomorphic to $\Gamma(A, T(A)) *|T(A)-T(K):|$, and this accounts for the generators and nonamalgamating relations of the presentation.

Since $T(K)$ extends $T(A)$ for each $A \in \lambda_{1}$ the amalgamated subgroups will be homomorphic images of $\Gamma(A, T(A))$. Suppose now that $B \in \lambda_{0}$ is such that $A \subset B$. Once basepoints $v(A), v(B)$ are chosen for $A$ and $B$ we have a preferred set of generating loops for $\Gamma(A, T(A))$ and $\Gamma(B, T(B))$ using $T(A)$ and $T(B)$ respectively. By the inclusion homomorphism between these two we mean the one which assigns to each of the generating loops of $\Gamma(A, T(A))$ the one in $\Gamma(B, T$ (B)) represented by the path in $T(B)$ from $v(B)$ to $v(A)$, then the loop in $\Gamma(A, T$ (A)) followed by the path in $T(B)$ from $v(A)$ to $v(B)$. The description of the homomorphism from $\Gamma(A, T(A))$ to $\Gamma(B, T(B)) * \mid T(B)-T(K)$ : $\mid$ as given in the statement of the theorem then follows from Theorem 1.4 and the third condition of Proposition 2.2. This completes the proof.

Thus a presentation for the group of $K$ can be written down from the graph $W_{1}$ by merely picking a tree $T\left(W_{1}\right)$ and using the groups of the cover and the homomorphisms induced by inclusion. For applications this method requires little more effort than the standard form of van Kampen's theorem, but provides much more flexibility in decomposing the space whose fundamental group is desired. (For a simple example, consider dividing a torus into two cylinders as opposed to a torus with a disc removed and a disc.)

From an algebraic point of view there is an additional comment that should be made about Theorem 2.3. We are accustomed to the functor $\pi_{1}$ linking certain topological constructions with algebraic constructions; for example cartesian products with direct products, wedge products with free products, and certain covers with limits of group systems. What is the linkage in the case of a 1-stage cover?

Presentations of the form given by Theorem 2.3 do arise in combinatorial group theory. In this context the 1-stage cover gives rise to an HNN group whose base group is a tree product (cf. [3] for definitions). More recently, Serre has considered this type of algebraic construction more explicitly [5]. He defines a 
graph of groups as a graph whose vertices correspond to groups and whose edges correspond to amalgamated subgroups of the incident vertices. He then proceeds to construct a group which he calls the fundamental group of a graph of groups. Given a 1-stage cover $\Sigma$, its graph $W_{1}$ is easily viewed as such a graph of groups. Indeed $\Sigma$ is merely a geometrical realization of Serre's construction. With this terminology, the theorem becomes

Corollary 2.4. Let $\Sigma$ be a 1-stage cover of $K$. Then the fundamental group of $K$ is the fundamental group of the graph of groups determined by $\Sigma$.

3. Having concluded our discussion of the particular case represented by a 1stage cover, we now turn our attention to its natural extension, the $n$-stage cover. The approach will closely follow the ideas presented for 1-stage covers. In the definition that follows, the most restrictive condition other than that of finite levels of intersection is the third. As in the case of 1-stage covers this is necessary to insure that the algebraic information not available in the groups of the members of the cover is present explicitly in the graph of the cover. We now begin with the natural generalization of Definition 2.1 .

Definition 3.1. An $n$-stage cover $\Sigma$ of the complex $K$ consists of a finite nonempty sequence $\lambda_{0}, \ldots, \lambda_{n}$ of collections of subcomplexes of $K$ such that:

(i) $\lambda_{0}$ is a cover of $K$ by connected subcomplexes of $K$.

(ii) $\lambda_{i}$ is the set of all path components of pairwise intersections $A_{1} \cap A_{2}$, where $A_{1}, A_{2} \in \lambda_{i-1}$.

(iii) If $A \in \lambda_{i}$, then $A-\cup \lambda_{i+1}$ is connected and nonempty.

(iv) The set of path components of $\cup \lambda_{n}$ is equal to $\lambda_{n}$ (or equivalently, $\lambda_{n}$ consists of disjoint sets).

For convenience, we write $\Sigma=\bigcup_{i=0}^{n} \lambda_{i}$.

In the case of the 1-stage cover it was found that additional information was required in the form of the graph $W_{1}$; a similar situation is provided for now.

Definition 3.2. The graph $W(\Sigma)$ of an $n$-stage cover $\Sigma=\cup_{i=0}^{n} \lambda_{i}$ is a sequence of bipartite graphs $W_{1}, \ldots, W_{n}$ such that

(i) For each $i, W_{i}$ has one class of vertices in one-to-one correspondence with the members of $\lambda_{i-1}$ and the other in one-to-one correspondence with the path components of $\cup \lambda_{i}$.

(ii) For each $i, W_{i}$ has an edge between vertices in these two classes if and only if the subcomplexes of $K$ which correspond to these vertices have nonempty intersection.

The essential idea of the construction of a maximal tree for $K$ will be to choose maximal trees in each path component of $U \lambda_{i}$ which extend maximal trees already chosen for the path components of $\cup \lambda_{i+1}$. As in the case of a 1-stage cover the graph $W_{i+1}$ will govern the extension at that stage. Indeed the analogy with the case of the 1-stage cover can be made more exact. Some notation must be set first. 
Let $L$ be a path component of $U \lambda_{i}$ which determines a subcollection of $\lambda_{i}, \lambda_{i}^{\prime}$. Let $X$ be the component of $W_{i+1}$ which contains the vertices corresponding to the members of $\lambda_{i}^{\prime}$. For each $A \in \lambda_{i}^{\prime}$, let $A^{\prime}$ be the subcomplex which is the union of $A$ and those path components of $\cup \lambda_{i+1}$ which meet $A$. Let $\partial_{0}$ be the collection of $A^{\prime}$ for $A \in \lambda_{i}^{\prime}$, and let $\partial_{1}$ be the collection of path components of $U \lambda_{i+1}$ that correspond to vertices in $X$. It then follows that

Proposition 3.3. $\Delta=\partial_{0} \cup \partial_{1}$ is a 1-stage cover of the path component $L$, and the graph of $\Delta$ is isomorphic to $X$.

Proof. That $\partial_{0}$ is a cover of $L$ by connected subcomplexes is clear by its definition. Similarly the members of $\partial_{1}$ are all disjoint. Hence the path components of the pairwise intersection of members of $\partial_{0}$ will be members of $\partial_{1}$ by the way that the $A \in \lambda_{i}^{\prime}$ were enlarged to the $A^{\prime}$ of $\partial_{0}$. Since for $A^{\prime} \in \partial_{0}, A^{\prime}-\cup \partial_{1}$ is just $A-\cup \lambda_{i+1}, A^{\prime}-\cup \partial_{1}$ will be nonempty and connected. The construction also insures that the graph of $\Delta$ is isomorphic to $X$. So the proof is complete.

Thus a maximal tree $T$ can be chosen for $K$ by a repeated series of applications of Proposition 2.2. The construction proceeds by choosing maximal trees in each path component of $U \lambda_{i}$ using the above proposition which presents these path components as 1-stage covers. Of course when Proposition 2.2 is applied the trees chosen at the preceding stage are used to initiate the construction. In any cases where a path component of $\cup \lambda_{i}$ is itself a member of $\lambda_{i}$ disjoint from the forest constructed up to that point any maximal tree can be chosen. The reader is reminded that in addition to selecting a maximal tree for $K$, this process will provide a correspondence between edges in the $W_{i+1}$ of $W(\Sigma)$ and edges in $A-\cup \lambda_{i+1}$ for $A \in \lambda_{i}$.

Again applying the results of $\S 1, \Gamma(K, T)$ can be considered as the limit of a group system involving the groups $\Gamma(A, A \cap T)$ and the induced homomorphisms: $k_{A B}: \Gamma(A, A \cap T) \rightarrow \Gamma(B, B \cap T)$ for $A, B \in \Sigma$ such that $A \subset B$. As was the case with 1-stage covers the object now is to use the graph of $\Sigma$ to describe this system in terms of groups $\Gamma(A, T(A))$ and homomorphisms between them. In what follows we shall make free use of the correspondence that exists between edges in the graph of $\Sigma$ and edges in the members of $\Sigma$. If one keeps in mind that edges in an $A \in \lambda_{i}$ are being considered on the basis of the properties of the corresponding edges in $W_{i+1}$ little confusion should arise.

Let $A \in \Sigma$. We know that $\Gamma(A, A \cap T) \approx \Gamma(A, T(A)) *|T(A)-(A \cap T):|$ for any maximal tree $T(A)$ extending $A \cap T$. The idea in finding such a $T(A)$ will be to recall that the construction of $A \cap T$ has been done by the extension of maximal trees chosen in subcomplexes of $A$. At certain stages edges had to be deleted, and $T(A)$ can be obtained by adding some of them back in. Since the $T\left(W_{i}\right)$ chosen in the $W_{i}$ determined the deletions, they and the $W_{i}$ can determine the additions. To this end in each $W_{i}$ consider the subgraph that is determined by edges of the form $(\bar{B}, \bar{C})$ where $C$ is a subcomplex of $A$. Let this subgraph be denoted by $W_{i}(A)$ (it need not be connected). Now $T\left(W_{i}\right) \cap W_{i}(A)$ can be 
extended to a maximal forest in $W_{i}(A)$ by the addition of a collection of edges $\bar{D}_{i}(A)$. Let $D_{i}(A)$ be the corresponding collection of edges in $A$. Then if $A \in \lambda_{i}$ the claim is that $D(A)$, the union of the $D_{j}(A)$ for $i+1 \leq j \leq n$, will provide the desired extension of $A \cap T$. The proof of this fact must be preceded by a lemma.

Lemma 3.4. Let $C$ be the subcomplex of $A \in \lambda_{j}$ which corresponds to a component $G$ of the graph $W_{i}(A)$ for some $i>j+1$. Then there exists a unique (reduced) path in $C \cap T(A)$ between any two vertices of $C$.

Proof. The proof will proceed by induction on $i$ beginning with $i=n$ and working towards $i=0$. The basis and inductive steps are quite similar and will be done simultaneously. Let the vertices $v_{1}, v_{2}$ be given. Then $v_{1}, v_{2}$ correspond uniquely to vertices of $G$. (If $v_{k}$ belongs to $B \in \lambda_{i}$ associate $v_{k}$ with the vertex corresponding to the component containing $B$, otherwise associate $v_{k}$ with the $\bar{B}$ of the $B$ such that $v_{k} \in B-\cup \lambda_{i}$.) Consider the unique path $\bar{p}$ which is given in the maximal tree of $G$ between the associated vertices $\bar{v}_{1}$ and $\bar{v}_{2}$. The edges of $\bar{p}$ give rise to a sequence of edges of $C \cap T(A)$ which will be expanded to the desired path. Two types of gaps have to be bridged corresponding to the two classes of vertices of $G$. For a vertex $\bar{B}$ with $B \in \lambda_{i-1}$ we need a unique path in $B \cap T(A)$ between any two vertices of $B-\cup \lambda_{l}$. This is assured by the way that the maximal tree is chosen for the $\partial_{0}$ elements of the 1-stage cover which $B-U \lambda_{i}$ was part of during some stage of the construcion of $T$ for $K$. Now consider the other type of vertex. For a unique path between any two vertices in $B \cap T(A)$ for a $B$ which is a component of $\cup \lambda_{i}$ two cases must be considered. If $i=n$, our basis case, then $B \cap T(A)=B \cap T$ is itself a maximal tree in $B$. In the case that $i \neq n$ we use the induction hypothesis for $i+1$. The resulting path so obtained from $v_{0}$ to $v_{1}$ must be unique otherwise one of the unique path properties in $W_{i}(A), B-U \lambda_{i}$, or $B$ a component of $U \lambda_{i}$ will be contradicted. This completes the proof of the lemma.

We can now obtain

Proposition 3.5. For $A \in \Sigma, T(A)=D(A) \cup(A \cap T)$ is a maximal tree in $A$ which extends $A \cap T$.

Proof. Suppose $A \in \lambda_{i}$; pick a vertex $v_{0} \in A-\cup \lambda_{i+1}$. To show that $T(A)$ is a maximal tree in $A$ it suffices to show that there is a unique path in $T(A)$ from $v_{0}$ to any other vertex $v \in A$. Note that once edges from $D_{i+1}(A)$ are added to $T \cap A^{\prime}$ a maximal tree for $A^{\prime}$ is obtained. This provides for a unique path from $v_{0}$ to any other $v$ in $A-U \lambda_{i+1}$. Now let $v$ be in some component $C^{\prime}$ of $U \lambda_{i+1}$ whose intersection with $A$ is $C$. Let $v^{\prime}$ be the unique vertex of $C$ determined by the edge $(\bar{C}, \bar{A})$. The desired path is to go to $v^{\prime}$ first and then to $v$ using Lemma 3.4. If the path is not unique a contradiction to either the lemma or the construction of the tree in $A^{\prime}$ occurs. This concludes the proof.

So for any $A \in \Sigma$ we have that $\Gamma(A, A \cap T) \approx \Gamma(A, T(A)) *|D(A):|$. Our attention now turns to the homomorphisms $k_{A B}: \Gamma(A, A \cap T) \rightarrow \Gamma(B, B \cap T)$ 
where $A \in \lambda_{i}, B \in \lambda_{j}$ are such that $A \subseteq B$. We wish to determine what the induced homomorphism

$$
\begin{aligned}
\bar{k}_{A B}: \Gamma(A, T(A)) *|D(A):| \stackrel{\varphi^{-1}}{\longrightarrow} \Gamma(A, A \cap T) \stackrel{k_{A B}}{\longrightarrow} \Gamma(B, E \cap T) \\
\stackrel{\Phi}{\longrightarrow} \Gamma(B, T(B)) *|D(B):|
\end{aligned}
$$

will be. As with the case of 1-stage covers $\bar{k}_{A B}$ can be related to a homomorphism induced by inclusion

$$
i_{A B}: \Gamma(A, T(A)) *|D(A):| \rightarrow \Gamma(B, T(B)) *|D(B):| \text {. }
$$

The homomorphism $i_{A B}$ is determined given basepoints $v(A)$ and $v(B)$ in $A-U \lambda_{i+1}$ and $B-\cup \lambda_{j+1}$ respectively. Let $p$ be the unique path in $T(B)$ from $v(B)$ to $v(A)$. For $x \in \Gamma(A, T(A))$ let $\bar{x}$ be the representative $v(A)$ based loop using $T(A)$. Then $i_{A B}(x)$ is the element of $\Gamma(B, T(B)) * \mid D(B)$ : | represented by $p \bar{x} p^{-1}$. As for elements corresponding to members of $D(A)$ there are two cases. If the edge $\bar{y} \in \bar{D}(A)$ corresponding to $y \in D(A)$ is also a member of $T(B)$ $-B \cap T$ then $i_{A B}(y)$ is the corresponding member of $D(B)$. If not then because of the way $T(B)$ has been constructed the addition of $\bar{y}$ to $T(B)$ gives rise to a loop in $T(B)$. In this case $i_{A B}(y)$ is assigned to that element of $\Gamma(B, B(T))$. Since $i_{A B}$ is clearly a homomorphism in each factor we get the homomorphism

$$
i_{A B}: \Gamma(A, T(A)) *|D(A):| \rightarrow \Gamma(B, T(B)) *|D(B):| \text {. }
$$

The relation of $\bar{k}_{A B}$ to $i_{A B}$ is determined by the graph of the cover. The path $p$ consists of edges of $T$ and $D(B)$. We take $\alpha(B, A) \in D(B)$ to be the word determined by reading off these edges of $D(B)$ as they occur on $p$. Then $\alpha(B, A)$ can be read off from the graph of $\Sigma$ as follows. Each edge $e$ of $W_{i}(B)$ determines a component of $U \lambda_{i}$ and indeed one of its vertices is a member of that component. In the component of $W_{i+1}(B)$ let the vertex determined by the vertex of $e$ be marked. Then using the marked vertices to pass from $W_{i}(B)$ to $W_{i+1}(B), \alpha(B, A)$ will correspond to the sequence of edges in $W(B)$ from $\bar{B}$ to $\bar{A}$. The $\bar{k}_{A B}$ can now be given by.

Proposition 3.6. $\bar{k}_{A B}: \Gamma(A, T(A)) *|D(A):| \rightarrow \Gamma(B, T(B)) *|D(B):|$ is the composite of $i_{A B}$ followed by the inner automorphism given by the element $\alpha(B, A)$.

Proof. For those generators of $\Gamma(A, T(A)) *|D(A):|$, considered as edges of $A$, that have the property that the paths in $T(A)$ from $v(A)$ to their initial and terminal vertices lie also in $T(B)$ the proof follows as in the similar case for 1stage covers. The others will correspond to some edge in a component $C$ of $A-\cup \lambda_{i+1}$ with the property that $(\bar{C}, \bar{A})$ was used in $T(A)$ but not for $T(B)$. Let $t$ be the edge in $A$ corresponding to $(\bar{C}, \bar{A})$. To handle the case of such an $x$ in $C$ several paths will have to be considered explicitly. Let $q$ be the path in $T(A)$ from $v(A)$ to the initial vertex of $t$. Let $r$ be the path in $T(B)$ from the terminal 
vertex of $t$ to $v(A)$. Without loss of generality we assume that $q$ lies in $T(B)$ as well (the choice depends on the orientation of $t$ ). We also have paths $s_{1}, s_{2}$ lying in $T(A) \cap T(B)$ from the terminal vertex of $t$ to the initial and terminal vertices of $x$. The computation of $\bar{k}_{A B}(x)$ can now begin. For $x, \bar{x}=q t s_{1} x s_{2}^{-1} t^{-1} q^{-1}$ and as such $\varphi^{-1}(x)=t x t^{-1}$ in $\Gamma(A, A \cap T)$. Hence $k_{A B}\left(\varphi^{-1}(x)\right)=t x t^{-1}$ in $\Gamma(B, B$ $\cap T)$ so it remains to compute $\varphi\left(t x t^{-1}\right)$ in $\Gamma(B, T(B)) *|D(B):|$. In $\Gamma(B, B$ $\cap T), t$ is represented by $p q r^{-1} p^{-1}$ and $x$ is represented by $p r s_{1} x s_{2}^{-1} r^{-1} p^{-1}$, hence $t x t^{-1}$ is represented by $p q t s_{1} x s_{2}^{-1} t^{-1} q^{-1} p^{-1}$. Since $q t s_{1} x s_{2}^{-1} t^{-1} q^{-1}$ is the representative loop for $i_{A B}(x)$ we have that $\bar{k}_{A B}(x)=\alpha(B, A) i_{A B}(x) \alpha(B, A)^{-1}$ as desired. This completes the proof.

The results of Proposition 3.5 and 3.6 can now be summarized in the following theorem.

Theorem 3.7. Let $\Sigma$ be an $n$-stage cover of $K$. Then given a selection of maximal trees in the $W_{i}$ of $\Sigma$ and the $W_{i}(A)$ for each $A \in \Sigma$ there exist maximal trees $T$ for $K, T(A)$ for $A \in \Sigma$ such that $\Gamma(K, T)$ is the limit of a group system involving the groups $\Gamma(A, T(A)) *|D(A):|$ and homomorphisms $\bar{k}_{A B}$ which are the inclusion homomorphisms $i_{A B}$ followed by the inner automorphisms of the elements $\alpha(B, A)$.

Proof. The proof follows from the construction of $T$ and the explicit computations of $\Gamma(A, A \cap T), \Gamma(B, B \cap T)$, and $\bar{k}_{A B}$ made in Propositions 3.5 and 3.6.

Although the complexity of Theorem 3.7 renders it questionable as a computational algorithm for larger values of $\boldsymbol{n}$ it does answer our algebraic concerns as to the relation between the fundamental group of a complex and the fundamental groups of the members of a cover of that space. As for the relation being exact we have the following

Corollary 3.8. If $\Sigma$ is an $n$-stage cover of $K$ such that all the members of the graph of $\Sigma$ are homotopic to a point then the fundamental group of $K$ is isomorphic to the limit of a group system involving the fundamental groups of the members of $\Sigma$ and the inclusion homomorphisms between them.

Proof. The proof is given by noting that in this case all the $D(A)$ will be empty and the $\alpha(B, A)=1$.

As for the cases anticipated by the exact sequence at the end of $\$ 1$ where the strong relation above is not possible we can now say the following:

Corollary 3.9. Let $\Sigma$ be an $n$-stage cover of $K$. Then $\Gamma(K, T)$ is a semidirect product of $N(\Sigma, T)$ by $G(\Sigma, T)$ where $N(\Sigma, T)$ is the normal subgroup generated by the union of the $D(A)$ for $A \in \lambda_{0}$ and $G(\Sigma, T)$ is the limit of a group system involving the groups $\Gamma(A, T(A))$ and inclusion homomorphisms between them.

Proof. From \$1 we have the short exact sequence:

$$
1 \rightarrow N(\Sigma, T) \rightarrow \Gamma(K, T) \rightarrow G(\Sigma, T) \rightarrow 1 .
$$

From Proposition 3.5 we see that $W(T)=\cup D(A)$ as $A$ ranges over $\lambda_{0}$. Using 
the computation of the $\bar{k}_{A B}$ above the factor group $G(\Sigma, T)$ will be the limit of the group system involving the $\Gamma(A, T(A))$ and the $i_{A B}$. Using a similar computation for the $k_{A}: \Gamma(A, T \cap A) \rightarrow \Gamma(K, T)$ and the universal property of the limit of a group system a splitting homomorphism $\eta: G(\Sigma, T) \rightarrow \Gamma(K, T)$ can be constructed. This concludes the proof.

The interpretation of Corollary 3.9 is that a cover decomposes that portion of the algebraic information of the complex which the fundamental group represents. In terms of the cover that information is then represented locally in terms of the fundamental groups of the members and globally in terms of the way that these members are pieced together. In the case of an $n$-stage cover, a presentation for the fundamental group of the complex can be found in which the final combination of these two aspects is represented algebraically.

\section{REFERENCES}

1. J. C. Chipman, Subgroups of free products with amalgamated subgroups: $A$ topological approach, Trans. Amer. Math. Soc. 181 (1973), 77-87.

2. R. H. Crowell, On the van Kampen theorem, Pacific Math. 9 (1959), 43-50. MR 21 \#3849.

3. A. Karrass and D. Solitar, The subgroups of a free product of two groups with an amalgamated subgroup, Trans. Amer. Math. Soc. 150 (1970), 227-255. MR 41 \#5499.

4. L. Neuwirth, The algebraic determination of the genus of knots, Amer. J. Math 82 (1960), 791-798. MR 22 \# 11397.

5. J. Serre, Groups discrets, Notes written at Collège de France, 1968/69.

6. H. Seifert, Konstruktion dreidimensionaler geschlossen Räume, Ber. Verh. Sächs. Akad. Wiss. 83 (1931), 26-66.

7. C. H. Spanier, Algebraic topology, McGraw-Hill, New York, 1966. MR 35 \# 1007.

8. E. R. van Kampen, On the connection between the fundamental groups of some related spaces, Amer. J. Math. 55 (1933), 261-267.

Department of Mathematics, OAKLAND University, Rochester, Michigan 48063 